\title{
Efeito clínico do uso de probióticos como adjuvante ao tratamento periodontal não cirúrgico da periodontite: revisão sistemática
}

\author{
Clinical effect of the use of probitics as an adjuvant to the non-surgical periodontal treatment of \\ periodontitis: systematic review \\ Efecto clínico del uso de probióticos como adyuvante del tratamento periodontal no quirúrgico de \\ la periodontitis: revisión sistemática
}

\section{Resumo}

O presente estudo avaliou o efeito do uso dos probióticos como adjuntos à raspagem e alisamento radicular (RAR) no tratamento da periodontite. Foi realizada busca nas bases de dados eletrônicas PubMed e Cochrane, empregando como critérios de inclusão estudos clínicos randomizados e controlados, na língua inglesa, publicados até fevereiro de 2021. A administração dos probióticos como adjunto ao tratamento periodontal básico revela proveitos clínicos concentrados na redução do processo inflamatório, ganho clínico de inserção e redução na profundidade de bolsas periodontais, no índice de placa e sangramento à sondagem. Dessa forma, a diminuição de bolsas periodontais sugere menor risco de progressão da doença periodontal. O uso adjunto RAR + probióticos sugere um efeito benéfico em relação aos parâmetros clínicos, como profundidade de sondagem, nível clínico de inserção e sangramento à sondagem, especialmente com relação à diminuição de bolsas periodontais residuais. No entanto, há uma necessidade de ensaios clínicos de longo prazo, com maior variedade nas cepas probióticas utilizadas, para uma melhor elucidação acerca do papel terapêutico dos probióticos no tratamento da periodontite.

Palavras-chave: Doenças periodontais; Raspagem dentária; Probióticos.

\begin{abstract}
The present study analyzed the effect of using probiotics as adjuncts to scaling and root planing (SRP) in the treatment of periodontits. A search was performed in the electronic databases PubMed and Cochrane, using randomized controlled clinical studies in English, published until February 2021, as inclusion criteria. The administration of probiotics as an adjunct to basic periodontal treatment reveals clinical benefits concentrated in the reduction of the inflammatory process, clinical attachment levels and reduction in the depth of periodontal pockets, in the plaque index and bleeding on probing. Thus, the decrease in periodontal pockets suggests a lower risk of progression of periodontal disease. The SRP + probiotic adjunct use suggests a beneficial effect in relation to clinical parameters, such as probing depth, clinical attachment levels and bleeding on probing, especially in relation to the reduction of residual periodontal pockets. However, there is a need for long-term clinical trials, with greater variety in the probiotic strains used, for a better understanding of the therapeutic role of probiotics in the treatment of periodontits.
\end{abstract}

Keywords: Periodontal diseases; Dental scaling; Probiotics.

\section{Resumen}

El presente estudio analizó el efecto del uso de probióticos como coadyuvante del raspado y alisado radicular RAR en el tratamiento de la periodontitis. Realizado en búsqueda de bases de datos electrónicas PubMed y Cochrane, 
utilizando como criterio de inclusión estudios clínicos aleatorizados y controlados, en inglés, publicados hasta febrero de 2021. La administración de probióticos como complemento del tratamiento periodontal básico revela beneficios clínicos centrados em la reducción del proceso inflamatorio, la ganancia de inserción clínica y la reducción de la profundidad de las bolsas periodontales, el índice de placa y el sangrado al sondaje. Por tanto, la disminución de las bolsas periodontales sugiere un menor riesgo de progresión de la enfermedad periodontal. El uso adjunto de probióticos + RAR sugiere un efecto beneficioso en relación con parámetros clínicos como la profundidad del sondaje, el nivel de inserción clínica y el sangrado al sondaje, especialmente con respecto a una disminución de las bolsas periodontales residuales. Sin embargo, es necesario realizar ensayos clínicos a longo plazo, con una mayor variedad de cepas de probióticos utilizados, para dilucidar mejor el papel terapéutico de los probióticos en el tratamiento de la periodontitis.

Palabras clave: Enfermedades periodontales; Raspado dental; Probióticos.

\section{Introduçãa}

A doença periodontal (DP) afeta mais de $50 \%$ da população mundial e, apesar dos avanços científicos alcançados, ainda é considerada um problema de saúde pública global (Batchelor, 2014). É uma doença crônica, multifatorial, iniciada por microrganismos bacterianos que produzem reações infecciosas e inflamatórias, afetando o corpo local e sistematicamente (Williams, 1990). Tais espécies bacterianas apresentam-se na forma de biofilme disbiótico e, embora o fator etiológico primário da doença periodontal seja o biofilme bacteriano, a resposta do hospedeiro e a presença de doenças sistêmicas parecem estar associadas com o seu desenvolvimento (Williams, Offenbacher, 2000).

Clinicamente, a DP vai apresentar em seu estágio inicial, também chamado de gengivite, alteração de cor e aspecto da gengiva, edemas, sangramento à sondagem e caso não seja tratada, vai evoluir para um estágio mais avançado. A periodontite é caracterizada por dano extenso às fibras, migração apical do epitélio juncional a partir da junção cemento-esmalte, manifestações disseminadas de danos inflamatórios e imunopatológicos aos tecidos, podendo ter a perda do osso alveolar. A lesão não é mais localizada e o infiltrado de células inflamatórias se estende para o tecido conjuntivo (Mayanagi et al., 2009).

O tratamento convencional da DP inclui a ruptura mecânica do biofilme através da terapia periodontal não cirúrgica (NSPT), incluindo raspagem e alisamento radicular, educação em saúde bucal e instrução de higienização oral (Cobb, 2002). Entretanto, devido às limitações no processo de alisamento e raspagem radicular, a recolonização bacteriana representa um grande problema no restabelecimento da saúde periodontal. Por isso, tem se ampliado a busca por terapias alternativas que possibilitem o equilíbrio no número de bactérias e a expressão dos mediadores da inflamação, e consequentemente, melhorem os resultados do tratamento periodontal convencional (Vives-Soler, Chimenos-Küstner, 2020).

A ocorrência de efeitos colaterais, como o aumento da resistência dos patógenos e a redução da percepção do paladar humano, associados às terapias alternativas comumente sugeridas, a exemplo, o uso de farmacologia antimicrobiana sistêmica, fomentam um maior interesse científico no uso dos probióticos como uma nova estratégia de prevenção de doenças bucais (Esteban-Fernández et al., 2019).

Historicamente, em 1908, Elie Metchnikoff sugeriu o uso de bactérias ácido-lácticas como benéfica à saúde, apontando que a modificação da flora intestinal através da introdução de "micróbios úteis" poderia combater processos de autointoxicação e o envelhecimento dos tecidos intestinais (Guias Mundiais da WGO, 2011). O termo "probiótico" foi introduzido pela primeira vez em 1965 por Lilly e Stillwell e em 1989, Roy Fuller destacou que, para ser considerado probiótico, o microrganismo devia estar presente em estado viável, e introduziu a ideia de ter um efeito benéfico para o hospedeiro (Hao, Dong, Wu, 2015).

No campo da ciência periodontal, os probióticos têm sido associados a mudanças no padrão de resposta inflamatória, atenuando-a e favorecendo a cicatrização dos tecidos periodontais. Somado a isto, as cepas probióticas parecem competir com agentes patológicos por sítios de adesão e nutrientes como também, têm sido exploradas sua capacidade de produção de moléculas potencialmente antimicrobianas como o ácido lático e a reuterina (Scannapieco, Gershovich, 2020). Estas 
propriedades coadunam com aparentes melhorias no curso das doenças periodontais, como a diminuição na profundidade de bolsas e ganho clínico de inserção.

Dessa forma, o objetivo deste trabalho é elaborar uma revisão sistemática sobre o tratamento da periodontite através do uso dos probióticos associado ao RAR.

\section{Metodologia}

O presente estudo seguiu orientações de acordo com o protocolo PRISMA (Preferred Reporting Items for Systematic Reviews and Meta-Analyses) (Moher, 2015). A seleção dos estudos, extração de dados e análise da qualidade metodológica foram realizadas por dois avaliadores (Sousa, G. A.; Oliveira, A. C. N.), previamente capacitados, de forma independente.

Nos casos de discordâncias entre os resultados encontrados, a avaliadora de minerva, (Borges; C. A.), fez a avaliação final, de forma imparcial e técnica.

\subsection{Pergunta Foco}

"Qual o benefício clínico do uso de probióticos como adjuvante ao RAR no tratamento não cirúrgico da periodontite?".

\subsection{Critérios de Elegibilidade}

Foram selecionados artigos originais de acordo com os seguintes critérios: estudos clínicos randomizados e controlados, publicados até fevereiro de 2021, artigos na língua inglesa que avaliassem o tratamento não cirúrgico da periodontite associado ao uso de probióticos e que considerasse profundidade de sondagem como variável primária ou secundária.

Os critérios de exclusão foram estudos realizados em animais, estudos realizados em humanos com comprometimento sistêmico, fumantes ou que fizeram uso de antibióticos, estudos que não utilizaram probióticos e/ou terapia periodontal não cirúrgica no tratamento, bem como cartas ao editor, revisões históricas da literatura e artigos não publicados.

\subsection{Estratégia de Busca}

A pesquisa foi realizada selecionando artigos publicados na literatura através das bases de dados MEDLINE (PubMed) e Cochrane Library, utilizando como estratégia de pesquisa as seguintes palavras-chave: (Periodontitis OR periodontal disease OR chronic periodontitis) AND (intervention OR non-surgical periodontal treatment OR non-surgical periodontal therapy OR scaling and root planning OR SRP OR periodontal therapy OR periodontal treatment) AND (probiotic OR probiotic therapy).

A estratégia de busca foi definida a partir do método PICOS, descrito na Tabela 1. 
Tabela 1 - Estratégia de busca para revisão sistemática através do método PICOS.

POPULAÇÃO (P): Pacientes adultos, sistemicamente saudáveis, diagnosticados com doença periodontal;

INTERVENÇÃO (I): Administração da bactéria probiótica por via oral;

COMPARAÇÃO (C): Entre o grupo teste (RAR + probiótico) e o grupo controle (RAR + placebo);

DESFECHO (O): Evolução dos parâmetros clínicos da doença periodontal;

TIPO DE ESTUDO (S): Ensaio clínico randomizado e controlado.

Fonte: Autores.

\subsection{Seleção dos Estudos}

A seleção dos estudos ocorreu em duas etapas (Figura 1). No primeiro momento, houve a leitura dos títulos e resumos, selecionando os que se enquadraram nos critérios de elegibilidade. A partir desses, foi realizada a leitura completa dos artigos, definindo quais fariam parte da revisão sistemática.

Figura 1 - Etapas da seleção dos estudos.

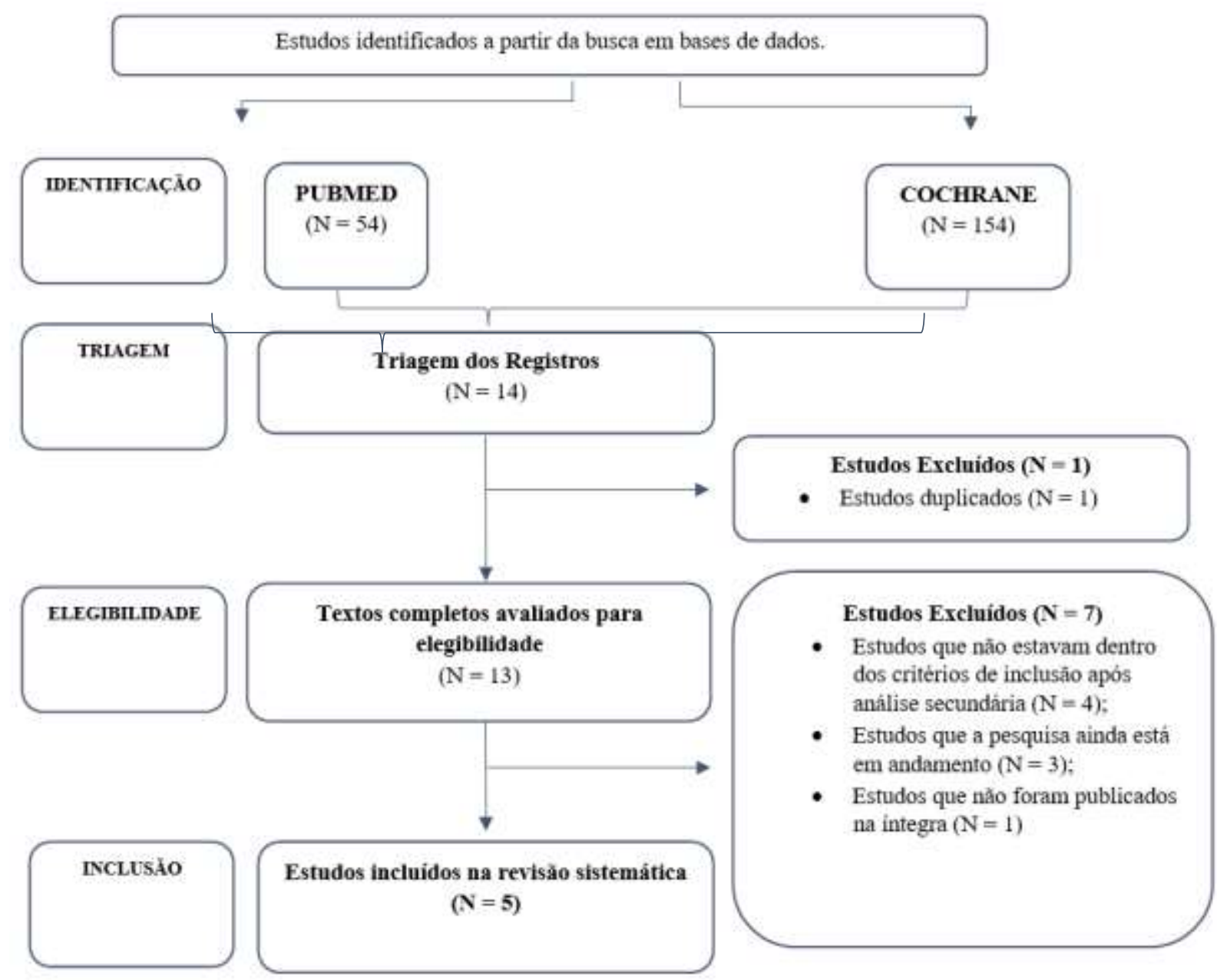

Fonte: Autores. 


\subsection{Extração dos Dados}

A extração dos dados foi realizada com o auxílio de uma tabela (Figura 2) com os seguintes dados: nome dos autores, tipo de estudo, critérios de inclusão, probiótico utilizado, frequência de administração e duração da terapia probiótica, acompanhamento, tratamento associado, efeitos adversos, parâmetros clínicos avaliados e principais conclusões.

Figura 2 - Resumo dos dados coletados dos ensaios clínicos randomizados incluídos na revisão sistemática.

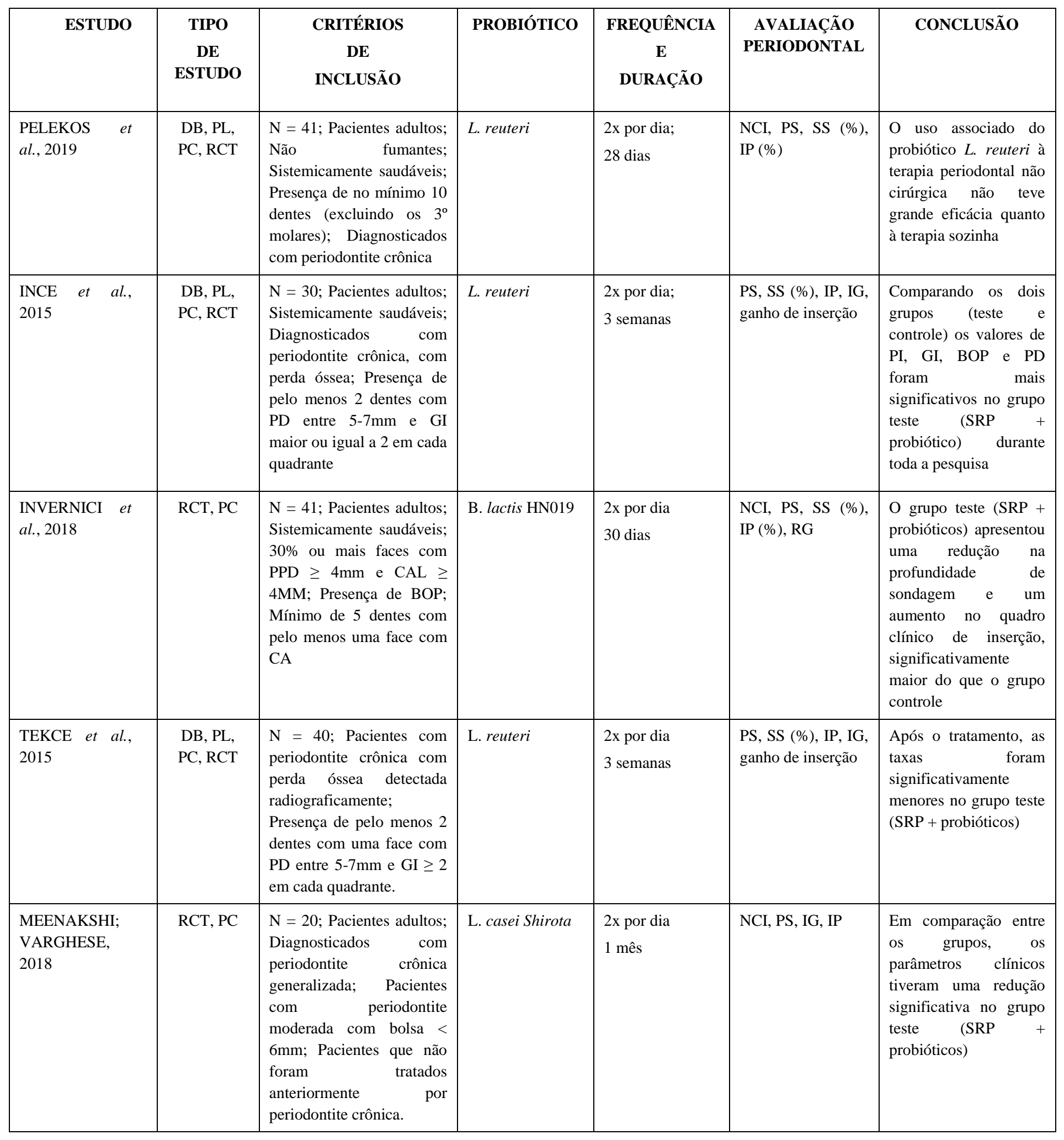




\subsection{Avaliação do Risco de Viés}

Os estudos incluídos na presente revisão foram avaliados de acordo com as recomendações da ferramenta The Cochrane Collaboration para avaliar o risco de viés em ensaios clínicos randomizados (Higgins, 2011). Tal avaliação concentrou-se nas seguintes divisões: randomização (viés de seleção), sigilo da alocação (viés de seleção), ocultamento de participantes da pesquisa e pesquisadores (viés de desempenho), ocultamento da avaliação de variáveis (viés de detecção), dados ausentes ou incompletos (viés de seguimento), síntese dos resultados (viés de relatório) e outros vieses.

O risco de viés de cada estudo foi categorizado de acordo com os seguintes critérios: alto risco de viés, baixo risco de viés ou risco incerto de viés, conforme demonstrado na Tabela 2.

Tabela 2 - Avaliação do risco de viés dos estudos incluídos. Em que, baixo risco de viés está representado por (-); alto risco de viés por (+) e risco incerto de viés por (?).

\begin{tabular}{|c|c|c|c|c|c|c|}
\hline ESTUDO & $\begin{array}{c}\text { RANDOMIZAÇÃO } \\
- \\
\text { VIÉS DE } \\
\text { SELEÇÃO }\end{array}$ & $\begin{array}{c}\text { SIGILO DA } \\
\text { ALOCAÇÃO } \\
- \\
\text { VIÉS DE } \\
\text { SELEÇÃO }\end{array}$ & $\begin{array}{c}\text { OCULTAMENTO DE } \\
\text { PARTICIPANTES DA } \\
\text { PESQUISA E } \\
\text { PESQUISADORES } \\
- \\
\text { VIÉS DE } \\
\text { DESEMPENHO }\end{array}$ & $\begin{array}{c}\text { OCULTAMENT } \\
\text { O DA } \\
\text { AVALIAÇÃo } \\
\text { DE VARIÁVEIS } \\
- \\
\text { VIÉS DE } \\
\text { DETECÇÃO }\end{array}$ & $\begin{array}{c}\text { DADOS } \\
\text { AUSENTES OU } \\
\text { INCOMPLETOS } \\
- \\
\text { VIÉS DE } \\
\text { SEGUIMENTO }\end{array}$ & $\begin{array}{c}\text { SÍNTESE DOS } \\
\text { RESULTADOS } \\
- \\
\text { VIÉS DE } \\
\text { RELATÓRIO }\end{array}$ \\
\hline $\begin{array}{l}\text { Pelekos et al., } \\
2019\end{array}$ & - & - & - & - & - & - \\
\hline Ince et al., 2015 & - & - & - & - & - & - \\
\hline $\begin{array}{l}\text { Invernici et al., } \\
2018\end{array}$ & - & + & - & - & - & - \\
\hline $\begin{array}{lll}\text { Tekce } & \text { et } & \text { al., } \\
2015 & & \end{array}$ & - & - & - & $?$ & - & - \\
\hline $\begin{array}{l}\text { Meenakshi; } \\
\text { Varghese, } 2018\end{array}$ & + & + & $?$ & $?$ & $?$ & - \\
\hline
\end{tabular}

Fonte: Autores.

\section{Resultados e Discussão}

\subsection{Seleção dos Estudos}

A busca sistemática resultou no total de 208 artigos potencialmente relevantes. Com a aplicação dos critérios de elegibilidade, 194 artigos foram excluídos com base no título e resumo. Após essa triagem, foi realizada a avaliação do texto completo dos 14 artigos selecionados, dentre os quais, 1 foi excluído por estar duplicado, 4 não estavam dentro dos critérios de inclusão após uma análise secundária, 1 ainda não estava publicado na íntegra e os 3 últimos, encontravam-se com a pesquisa em andamento. Por fim, 5 artigos foram incluídos nesta revisão sistemática.

\subsection{Características dos Estudos}

Todos os estudos são ensaios clínicos randomizados controlados (ECR), publicados na língua inglesa entre 2015 e 2019. 
Três cepas diferentes de Probióticos, administradas por via oral no formato de pastilhas em 4 dos 5 trabalhos e como bebida em 1 deles, foram utilizadas nos estudos, sendo o L. reuteri citado em 3 artigos, enquanto o B. lactis HNO19 e L. casei shirota em apenas 1 trabalho, cada. Em todos os artigos incluídos, há comparação entre grupos (grupo controle e grupo teste), com variações no período de acompanhamento entre 21 e 360 dias. Com relação ao número de participantes selecionados para compor a amostra, houve diferentes configurações, das quais, dois estudos incluíram 41 pacientes (Pelekos et al., 2019; Invernici et al., 2018), uma pesquisa selecionou 40 pacientes (Tekce et al., 2015), outro trabalho elegeu 30 pacientes (Ince et al., 2015) e o último, incluiu 20 pacientes (Meenakshi, Varghese, 2018).

\subsection{Risco de Viés}

A maioria dos estudos gerou sua sequência de alocação aleatória através do uso de programas de randomização computadorizada. Apenas Meenakshi, Varghese, 2018, utilizaram o método de jogar uma moeda. O trabalho teve seu risco de viés incerto no critério de ocultamento para participantes e pesquisadores acerca do grupo ao qual cada paciente pertencia, pois não descreve se houve quebra do sigilo da alocação em teste ou controle. Os demais artigos selecionados relatam que a revelação desta sequência só foi realizada após a finalização da análise dos resultados obtidos, inferindo a manutenção do ocultamento acerca da composição de cada grupo durante a realização das etapas anteriores. Ademais, a metodologia adotada para verossimilhança entre as intervenções placebo/controle foram narradas minuciosamente em quatro dos cinco artigos incluídos nesta revisão.

Em relação à intercorrências que pudessem ocasionar alteração no tamanho das amostras e/ou exclusão de dados das variáveis escolhidas por cada trabalho, observou-se preservação da quantidade de participantes em três dos estudos, explicitados sob formato de fluxograma (Figura 1). Pelekos et al., 2019 descreve perdas na amostragem total, justificando-as a partir da manifestação do interesse destes voluntários em abandonar sua participação no projeto, tendo como base alegações pessoais. Em Meenakshi, Varghese, 2018, não ocorreu perda dos participantes e nenhum deles reportou desconforto com a pesquisa.

\subsection{Variáveis Analisadas}

\subsubsection{Profundidade de Sondagem (PS) - Redução de Bolsas Periodontais}

Todos os estudos incluíram PS na avaliação clínica inicial e mostraram diferenças significativas na redução desta variável, como exposto na Figura 3. 
Figura 3 - Avaliação da redução de profundidade de sondagem em todos os estudos incluídos.

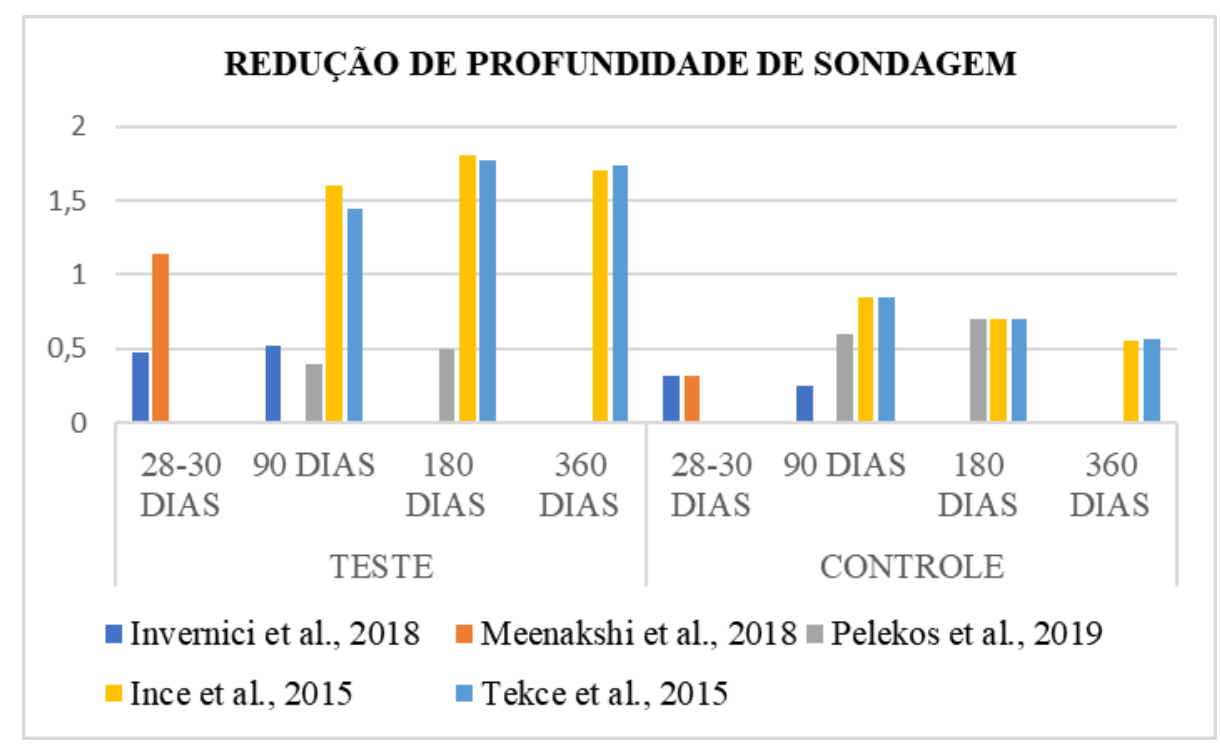

Fonte: Autores.

Segundo Pelekos et al., 2019, entre o grupo teste e o grupo controle, foram observadas mudanças nos valores encontrados, revelando uma diminuição na profundidade de sondagem no intervalo de 3 meses, com manutenção da mesma até o final dos 6 meses de pesquisa. No entanto, a diferença no valor de PS foi a mesma em ambos os grupos, $p<0.001$. Ince et al., 2015, houve uma maior redução de PS e maior ganho de inserção no grupo teste, ou seja, a favor do grupo que associou a RAR com os probióticos.

No estudo de Invernici et al., 2018, o grupo teste teve um grande ganho clínico de inserção e valores menores de PS quando comparado ao grupo controle no final dos 90 dias de pesquisa $(p<0,05)$. A análise dos resultados obtidos em bolsas com profundidade moderada e severa revelou-se favorável ao grupo teste. Comparando-se os valores percentuais de detecção de bolsas residuais de maior ou menor gravidade, após os 90 dias de pesquisa, a população teste apresentou em sua maioria, valores menores ou iguais a 3 milímetros, enquanto no grupo controle essa alteração de graduação foi menos expressiva.

Tekce et al., 2015, através da avaliação de profundidade, houve redução dos valores em ambos os grupos, entretanto, após 1 ano a diferença $(p<0.05)$ foi significantemente maior no grupo teste. Ao final do tratamento, Meenakshi, Varghese, 2018, fizeram a comparação entre os dois grupos e ambos tiveram a redução de $p<0.05$.

\subsubsection{Classificação das Bolsas Periodontais}

A profundidade das bolsas foi avaliada como um dos parâmetros clínicos em 3 dos 5 estudos incluídos. No entanto, devido a heterogeneidade presente nos artigos, a faixa em milímetros para classificação das bolsas, em moderadas ou profundas, não encontra-se padronizada. Desta maneira, houve dissonância entre os valores estabelecidos. Ince et al., 2015 subdividiram as bolsas em PS $\leq 5 \mathrm{~mm}$ e PS $\geq 5 \mathrm{~mm}$. Invernici et al., 2018, classificou as bolsas em 3 estágios sendo leves as que obtivessem PS $\leq 3 \mathrm{~mm}$, moderadas PS $\geq 4 \leq 6 \mathrm{~mm}$ e severas PS $\geq 7 \mathrm{~mm}$. Tekce et al., 2015 consideraram três grupos, sendo PS $\leq 4 \geq 5 \mathrm{~mm}$ nível baixo, PS $\geq 5 \mathrm{~mm}$ nível moderado e PS $\geq 5 \mathrm{~mm}$ nível alto.

\subsubsection{Nível Clínico de Inserção (NCI)}

O nível clínico de inserção foi adotado como variável primária em 3 dos 5 estudos incluídos nesta revisão. 
Pelekos et al, 2019 descreveram melhorias significativas nas taxas de NCI obtidas no intervalo de 3 meses (dia 90), com alterações mínimas até o prazo final (dia 180) As variações intergrupo não representaram diferenças estatisticamente relevantes $(p<0,2)$. Invernici et al., 2018, o grupo teste obteve maior ganho de inserção, o qual pontuou menor número de bolsas periodontais residuais moderadas ou profundas $(p<0,05)$. Meenakshi; Varghese, 2018, houve significativa melhora nas taxas de NCI no grupo teste decorrido 1 mês de intervenção. A comparação dos valores pós-tratamento entre os grupos controle e teste não pareado traduziu-se numa significância estatística $(p<0,05)$.

A avaliação do ganho de inserção está demonstrada na Figura 4.

Figura 4 - Avaliação do ganho de inserção em todos os estudos incluídos.

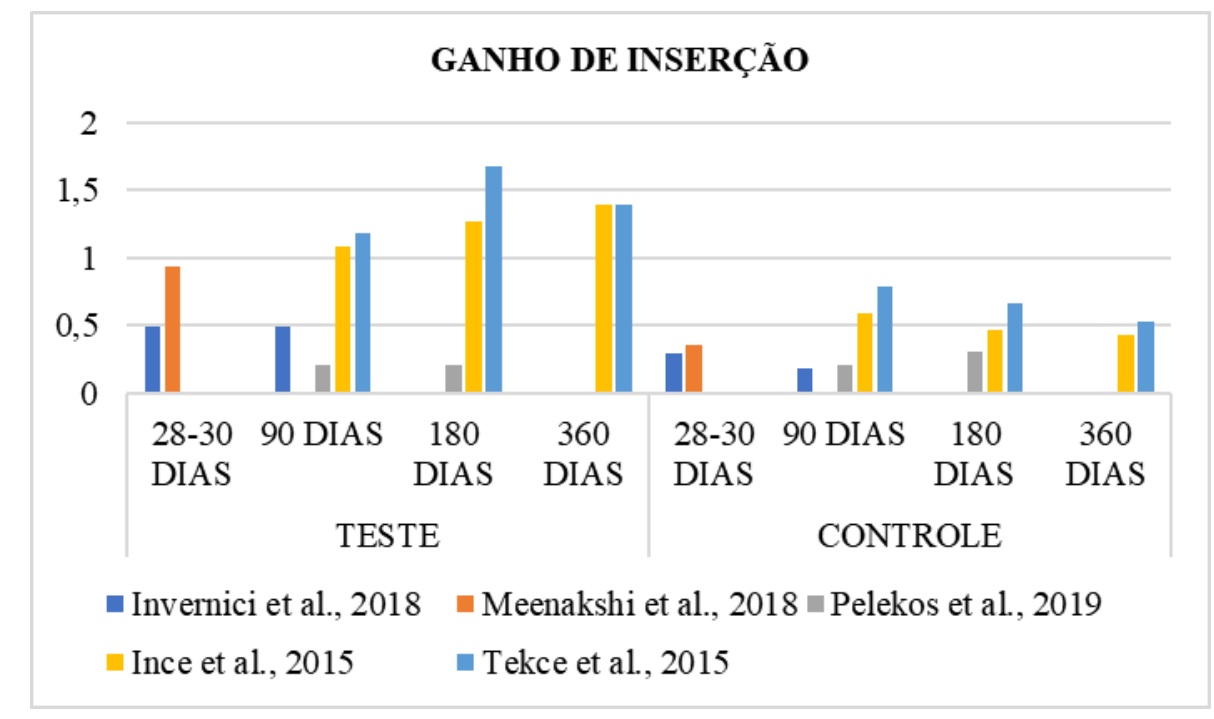

Fonte: Autores.

\subsubsection{Sangramento à Sondagem (SS)}

O sangramento à sondagem foi abordado como variável em 4 artigos dentre os 5 incluídos, sendo representado na Figura 5 . 
Figura 5 - Avaliação da redução de sangramento à sondagem em todos os estudos incluídos.

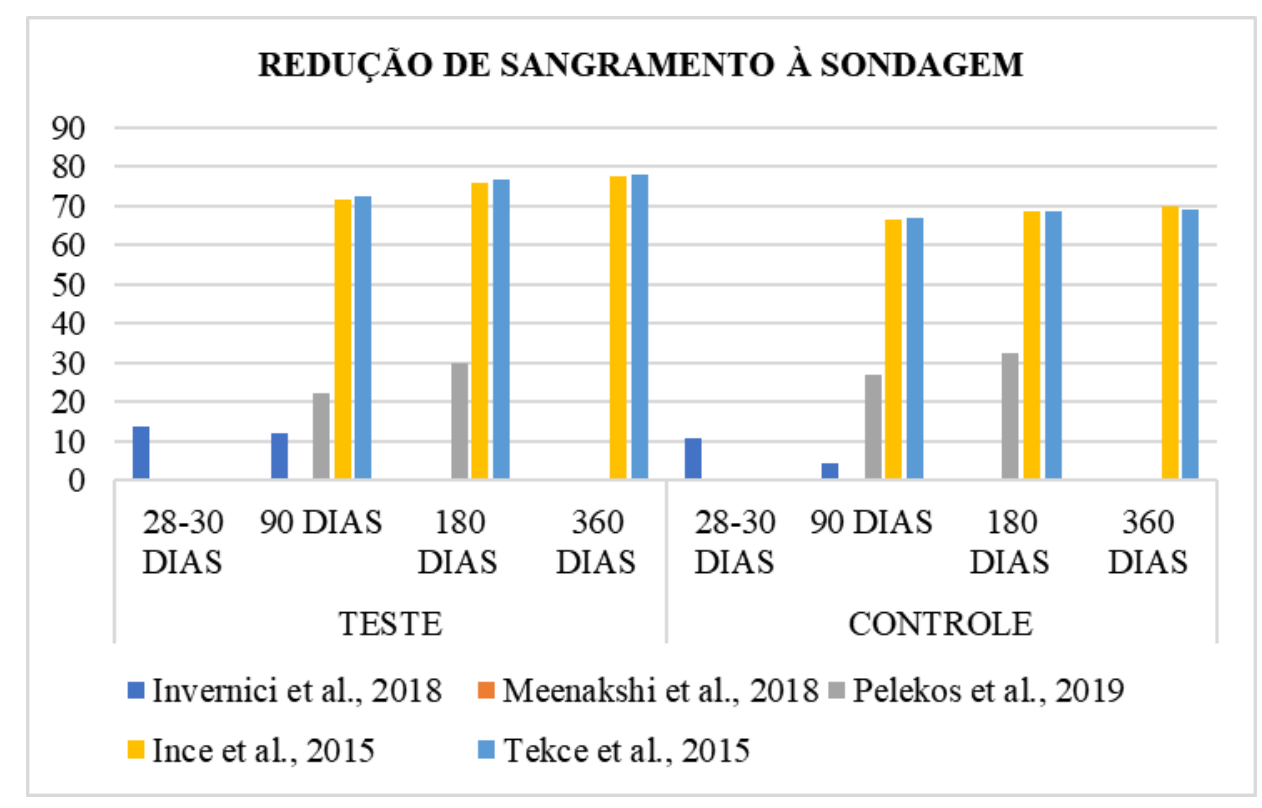

Fonte: Autores.

Pelekos et al., 2019, apontam presença de alterações significativas de melhora em ambos os grupos, nos primeiros três meses de acompanhamento e mudanças de menor relevância nos três últimos. A análise estatística constituiu-se de aproximadamente 50\% de redução nas taxas SS. Tal diferença, revelou-se de modo semelhante nas populações teste e controle, não havendo variações estatisticamente expressivas intergrupos. Ince et al., 2015, os índices de sangramento reduziram drasticamente no intervalo de tempo de 21 dias e manteve-se baixo pelo tempo restante de estudo nos dois grupos. Após a finalização do período de intervenção, as taxas de SS apresentadas pelo grupo teste se sobressaíram em relação ao grupo controle $(p<0.05)$. Invernici et al., 2018, descreve que o índice de sangramento reduziu de $30 \%$ para $18,80 \%$ entre o dia 0 e $90^{\circ}$ no grupo teste e de $35 \%$ para $30,71 \%$ do 0 para $90^{\circ}$ dia, no grupo controle.

Tekce et al., 2015, apresentam uma redução significativa datados os 21 dias do período intervencional e taxas dentro de um limiar baixo de maneira constante até o encerramento do acompanhamento. Ao final do estudo, a variável SS do grupo teste destacou-se em relação ao grupo controle $(p<0.05)$.

\subsection{5 Índice de Placa (IP)}

A variável índice de placa foi abordada em todos os trabalhos. A obtenção deste parâmetro foi descrita pelos autores (Pelekos et al., 2019; Tekce et al., 2015; Ince et al., 2015) como tendo sido realizada através do auxílio de uma sonda periodontal por um examinador calibrado no exame clínico inicial.

Pelekos et al., 2015, avaliaram esse parâmetro, obtendo uma redução de aproximadamente 55\% sem especificar o grupo ao qual este dado pertence.

Ince et al., 2015, relatam que os níveis de PI reduziram expressivamente no dia 21 do período intervencional. Após o tratamento, PI mostrou-se significativamente menor $(p<0.05)$ no grupo teste em relação ao controle, em todos os intervalos de tempo comparados.

Invernici et al., 2018, obtiveram o IP a partir da presença ou ausência de placa visível nos sítios avaliados. As taxas de IP apresentaram uma variação média de $23.85 \%$ para $21.65 \%$ no grupo teste, entre os dias 0 e $90^{\circ}$. Enquanto o grupo controle houve um aumento de $26.71 \%$ para $27.14 \%$ neste mesmo intervalo de tempo. 
A quantidade de biofilme visível foi incluída em apenas um artigo. Pelekos et al., 2019, trazem que ambos os grupos obtiveram uma diferença significativa $(p<0.001)$ e contínua observada no terceiro mês até o final do estudo.

\subsection{6 Índice Gengival (IG)}

O índice gengival aparece em três dos cinco estudos incluídos. Tekce et al., 2015; Ince et al., 2015, apontaram uma redução expressiva decorridos 21 dias de pesquisa, como também, a manutenção dessa alteração durante o tempo restante de ensaio clínico. Ao final do tratamento, esta diferença se mostrou de maior impacto no grupo teste. Meenakshi; Varghese, 2018, também obteve diferença significativa, de 2.08 para 0.89 , em favor do grupo teste ao final do estudo.

\subsubsection{Risco de Progressão da Doença Periodontal}

O risco de progressão da doença periodontal é mencionado em 2 dos 5 artigos incluídos. Ambos descrevem que a avaliação de risco de progressão é determinada pela presença de bolsas residuais.

Invernici et al., 2018, classificam o risco em baixo, moderado ou alto, mensurado após 90 dias de pesquisa. O grupo teste apresentou a média de $11 \%$ para risco leve, $3 \%$ para moderado e $6 \%$ para risco elevado. Enquanto o grupo controle apresentou média de $6 \%$ de risco leve, $4 \%$ moderado e $11 \%$ para risco elevado. Tekce et al., 2015, colocam que $25 \%$ dos pacientes do grupo teste obtiveram risco leve, $15 \%$ moderado e $60 \%$ riso elevado ao final do tratamento. Em contrapartida, o grupo controle teve $5 \%$ dos pacientes com risco baixo, $10 \%$ moderado e $85 \%$ risco elevado para a progressão da doença.

\section{Discussão}

A presente revisão sistemática teve como objetivo avaliar o benefício clínico do uso de probióticos como adjuvante no tratamento não cirúrgico da doença periodontal. A administração dos probióticos como adjunto ao tratamento periodontal básico revela proveitos clínicos concentrados na redução do processo inflamatório, ganho clínico de inserção e redução na profundidade de bolsas periodontais, como também no índice de placa e sangramento à sondagem (Ince et al., 2015).

O desenvolvimento e a progressão das doenças periodontais correlacionam-se com a resposta imunológica e mecanismos de feedback positivos e negativos para inflamação nos indivíduos acometidos. Desta maneira, justifica-se a busca por terapêuticas moduladoras destes processos, visando uma ação da RAR em favor do restabelecimento da saúde periodontal. Embora os mecanismos de ação probiótica ainda não se encontrem completamente esclarecidos, grande parcela dos estudos aponta para a sua capacidade de competição por superfícies de adesão e nutrientes. Tal capacidade pode ser compreendida como uma relação mutualista, pois a presença desses microrganismos favorecem a colonização por agentes patológicos (Hao, Dong, Wu, 2015).

Além de impactar na dinâmica complexa do microbioma existente nos tecidos periodontais, a composição probiótica mais comumente relatada, pertence aos gêneros Lactobacillus e Bifidobacterium, dentre os quais, destaca-se a espécie Lactobacillus reuteri, associada a produção de compostos potencialmente antimicrobianos, como a reuterina e o ácido lático (Tekce et at., 2015).

A observação terapêutica destas moléculas, indicam que o ácido lático seja capaz de penetrar a membrana plasmática bacteriana e alterar o pH do meio citoplasmático, acidificando-o e ocasionando consequentemente, a inibição da atividade do agente patológico. Enquanto a reuterina, tem sido descrita como um composto capaz de inibir o crescimento bacteriano por atuar como análogo da D-ribose, inibindo a ribonucleotídeo redutase, o que impede a formação de novos ribonucleotídeos para a síntese do DNA. Esta atividade inibitória foi relatada como presente frente a bactérias Gram-positivas e Gram-negativas, configurando um amplo espectro de ação fomentador de interesse clínico (Hao, Dong, Wu, 2015). 
Invernici et al., 2018, justificam a escolha pela utilização de cepas pertencentes ao gênero Bifidobacterium por seu possível efeito protetor contra a destruição óssea em estudos pré-clínicos (Ricoldi et al., 2017). Tal efeito pode ter sido traduzido na demonstração de benefícios adicionais nos parâmetros de PS e NCI, especialmente em bolsas profundas, ao final do tratamento no grupo que fez uso dos probióticos, em comparação ao grupo controle. O estudo revelou que bolsas consideradas moderadas PS $\geq 4 \leq 6 \mathrm{~mm}$ apresentaram redução significativa nos dois grupos avaliados, porém, com maior redução significativa no grupo teste, conferindo uma involução na doença periodontal mais representativa nesse grupo.

Além disso, o uso do probiótico possibilitou modulação na microbiota, com diminuição expressiva de bactérias patogênicas, como a Porphyromonas gingivalis. É possível que essa modulação seja dada através da adesão ao biofilme subgengival e mecanismos de competição por nutrientes e sítios de ligação. $\mathrm{O}$ grupo teste apresentou maiores taxas de bactérias comensais no biofilme subgengival quando comparado ao grupo controle no final dos 90 dias, tais bactérias são associadas à saúde periodontal e podem auxiliar o controle da inflamação dos tecidos periodontais. Além destas, o grupo teste apresenta menor proporção de bactérias anaeróbias Gram-negativas, espécies envolvidas na patogênese da doença periodontal (Hao, Dong, Wu, 2015).

Tekce et al., 2015, relatam que a utilização de probióticos teve um papel aparente no retardo da progressão da doença, atribuindo-a possivelmente a detecção da substância reuterina entre $21^{\circ}$ e $90^{\circ}$ dia. Ao final do estudo, o grupo teste obteve menor índice de bolsas periodontais profundas, menor risco de progressão da doença e menores taxas de indicação cirúrgica periodontal. Esse resultado pode ser atribuído aos efeitos do desbridamento mecânico do controle da placa supragengival e redução da contagem de células viáveis e proporção de anaeróbios obrigatórios.

A reuterina sugere atividade bactericida e bacteriostática contra uma ampla gama de bactérias patogênicas, leveduras, fungos, protozoários e vírus. Seu mecanismo de ação faz a inibição das espécies patogênicas através da indução do estresse oxidativo nas células. Entretanto, destaca-se que 6 meses após o período intervencional com probióticos (uso por 3 semanas), não foi detectada a presença do microrganismo probiótico nem de seu produto reuterina. Ademais, foi observada a recolonização por agentes patológicos (Tekce et al., 2015).

Apesar dos aparentes resultados favoráveis ao grupo teste, o estudo de Pelekos et al., 2019, demonstrou que estatisticamente não há diferenças significativas entre as populações teste e controle. Os autores relatam não ter evidências suficientes para apoiar o uso do probiótico L. reuteri como adjunto ao RAR. Dentre os estudos incluídos nesta revisão, os parâmetros clínicos periodontais avaliados no grupo teste de Pelekos et al., 2019, apresentaram menores reduções, as quais são compatíveis numericamente por resultados obtidos nos grupos controle dos demais estudos. Alguns fatores como, calibração do avaliador clínico, uso de guia de acrílico para padronizar o sítio de sondagem e experiência clínica do operador que executou a RAR, interferiram nos resultados clínicos, resultando em diferenças entre estudos.

A análise da redução da PS revelou melhora no estado de saúde periodontal para ambos os grupos em todos os estudos incluídos, no entanto, os valores obtidos manifestaram vantagens clínicas para as populações que fizeram uso dos probióticos. Ince et al., 2015, destacam que a redução dos marcadores inflamatórios no grupo teste em relação ao grupo controle pode ter sido traduzida no retardo da progressão da doença periodontal e com isto, melhora dos parâmetros clínicos de NCI e PS, sugerindo uma ação anti-inflamatória envolvendo os probióticos. A análise de parâmetros bioquímicos concentrouse na identificação dos níveis de MMP-8, TIMP-1 e GCF, indicando uma diminuição nos níveis de MMP-8 e aumento nas taxas de TIMP-1 para ambos os grupos $(\mathrm{p}<0.05)$. Entretanto, os resultados sugerem superioridade do grupo teste em todos os intervalos de tempo mensurados, com exceção do $360^{\circ}$ dia onde os níveis se enquadram nos seus valores basais para os dois grupos ( $\mathrm{p}>0.05)$.

As bolsas periodontais foram classificadas em leves, moderadas ou profundas em 3 dos 5 artigos. No entanto, não houve consenso entre os autores com relação à categorização da faixa em milímetros atribuída a cada nível de severidade. 
Apesar disto, nos três estudos, o grupo teste apresentou menor quantidade de bolsas profundas quando comparado ao grupo controle, sendo essa diferença estatisticamente significante. Sugerindo assim que o uso dos probióticos possibilitou redução da doença com maior ênfase. Para estudos futuros, sugere-se padronização da classificação de bolsas periodontais, fato que pode permitir comparação estatística em relação a esta variável.

Além da redução do processo inflamatório, a diminuição de bolsas periodontais sugere menor risco de progressão da doença periodontal. Tekce et al., 2015, demonstraram que, após 360 dias, $60 \%$ dos participantes do grupo teste e $85 \%$ do grupo controle possuíam um risco elevado de progressão da doença. Por outro lado, Invernici et al, 2018, observaram que, após 90 dias, $30 \%$ dos participantes do grupo teste e 52,4\% dos participantes do grupo controle demonstraram risco elevado de progressão da doença. As diferenças nos resultados dos dois estudos são observadas não apenas no tipo de probiótico utilizado, mas também no período de acompanhamento de cada estudo. O período de análise mais extenso permite avaliação dos resultados em longo prazo, porém pode permitir recolonização bacteriana e sugerir prazo reduzido de ação probiótica.

Ressalta-se que apesar dos aparentes resultados favoráveis às populações teste, nos 4 dos 5 estudos incluídos nesta revisão, a implementação de uma terapêutica encontra-se sujeita a diversos fatores que devem ser contabilizados para justificar sua aplicabilidade ou não ao tratamento convencional. Embora os probióticos tenham suas potencialidades destacadas, o custo adicional, adesão ao tratamento e o acesso dos pacientes ao mesmo, são alguns dos fatores que devem ser pontuados nos protocolos clínicos que os insiram como adjuntos a RAR.

\section{Conclusão}

O uso adjunto dos probióticos ao tratamento periodontal sugere um efeito benéfico em relação aos parâmetros clínicos, como profundidade de sondagem, nível clínico de inserção e sangramento à sondagem, especialmente, com relação a diminuição de bolsas periodontais residuais.

No entanto, a carência de ensaios clínicos de longo prazo, somada a necessidade de maior variedade nas cepas probióticas utilizadas, geram ressalvas para sua implementação de maneira difusa. Outros fatores além das potencialidades dos agentes probiótico devem ser mensurados para sua consolidação. Ressalta-se a necessidade de mais estudos, com rigor metodológico e baixo risco de viés para uma melhor elucidação acerca do papel terapêutico dos probióticos na periodontia.

\section{Referências}

Batchelor, P. (2014). Is periodontal disease a public health problem? Nat Publ Gr. 405-9.

Cobb, C. M. (2002). Clinical significance of non-surgical periodontal therapy: An evidence-based perspective of scaling and root planing. J Clin Periodontol. 29(SUPPL. 2):22-32.

Esteban-Fernández, A., Ferrer, M., Zorraquín-Peña, I., López-López, A., Moreno-Arribas, M., \& Mira, A. (2019). In vitro beneficial effects of Streptococcus dentisani as potential oral probiotic for periodontal diseases. J Periodontol. 90(11):1346-55.

Guias mundiais da WGO. (2011). Probióticos e prebióticos.

Hao, Q., Dong, B., \& Wu, T. (2015). Probiotics for preventing acute upper respiratory tract infections. Cochrane Database Syst Rev. (2).

Ince, G., Gursoy, H., Ipçi, S., Cakar, G., Emekli-Alturfan, E., \& Yilmaz, S. (2015). Clinical and Biochemical Evaluation of Lactobacillus Reuteri Containing Lozenges as an Adjunct to Non-Surgical Periodontal Therapy in Chronic Periodontitis. J Periodontol. 1-13.

Invernici, M., Salvador, S., Silva, P., Soares, M., Casarin, R., Palioto, D., Souza, S., Taba Júnior, M., Novaes Júnior, A., Furlaneto, F., \& Messora, M. (2018). Effects of Bifidobacterium probiotic on the treatment of chronic periodontitis: A randomized clinical trial. 1198-210.

Higgins, J., Altman, D., Gøtzsche, P., Jüni, P., Moher, D., Oxman, A., Savovic, J., \& Schulz, K. (2011). The Cochrane Collaboration's tool for assessing risk of bias in randomised trials. BMJ. 343(7829):1-9.

Mayanagi, G., Kimura, M., Nakaya, S., Hirata, H., Sakamoto, M., Benno, Y., \& Shimauchi, H. (2009). Probiotic effects of orally administered Lactobacillus salivarius WB21-containing tablets on periodontopathic bacteria : a controlled, randomized clinical trial. (3):506-13. 
Research, Society and Development, v. 10, n. 14, e149101421703, 2021

(CC BY 4.0) | ISSN 2525-3409 | DOI: http://dx.doi.org/10.33448/rsd-v10i14.21703

Meenakshi, S. S., \& Varghese, S. (2018). Adjunctive effect of probiotic (Lactobacillus casei Shirota) to scaling and root planing in the management of chronic periodontitis. 10(8):3-6.

Moher, D., Shamseer, L., Clarke, M., Ghersi, D., Liberati, A., Petticrew M., Shekelle, P., \& Stewart, L. (2015). Preferred reporting items for systematic review and meta-analysis protocols ( PRISMA-P). 2015 statement. 1-9.

Pelekos, G., Ho, S., Acharya, A., Leung, W., \& McGrath, C. (2019). A double-blind, paralleled-arm, placebo-controlled, randomized clinical trial of the effectiveness of probiotics as an adjunct in periodontal care. J Periodontol.

Ricoldi, M., Furlaneto, F., Oliveira, L., Teixeira, G., Pischiotini, J., Moreira, A., Ervolino, E., Oliveira, M., Bogsan, C., Salvador, S., \& Messora, M. (2017). Effects of the probiotic Bifidobacterium animalis subsp. lactis on the non-surgical treatment of periodontitis. A histomorphometric, microtomographic and immunohistochemical study in rats. PLoS One. 12(6):1-15.

Scannapieco, F., \& Gershovich, E. (2020). The prevention of periodontal disease - An overview. 9-13.

Tekce, M., Ince, G., Gursoy, H., Dirikan, S., Cakar, G., Kadir, T., \& Yilmaz, S. (2015). Clinical and microbiological effects of probiotic lozenges in the treatment of chronic periodontitis: a 1-year follow-up study. 363-72.

Vives-Soler, A., \& Chimenos-Küstner, E. (2020). Effect of probiotics as a complement to non-surgical periodontal therapy in chronic periodontitis: A systematic review. Med Oral Patol Oral y Cir Bucal. 25(2):e161-7.

Williams, R. C. (1990). Periodontal disease. The New England Journal of Medicine.

Williams, R. C., \& Offenbacher, S. (2000). Periodontal medicine: the emergence of a new branch of periodontology. Periodontol. 23(7):9-12. 